\title{
Overview on Implementation Planning of Energy Internet Based on Energy Hub
}

\author{
Jiali Liang ${ }^{1, a}$, Yanhong Luo ${ }^{1, b}$, Dongsheng Yang ${ }^{1, c}$, Xingqiang Guo ${ }^{2, d}$ \\ ${ }^{1}$ College of Information Science and Engineering, Northeastern University, Shenyang, \\ 110819, China \\ ${ }^{2}$ Shenzhen Electric Power Company Limited, Shenzhen, 518000, China \\ aemail: \\ 370681845@qq.com, bemail: luoyanhong@ise.neu.edu.cn, cemail: ds_yang@126.com, ${ }^{d} e m a i l:$ \\ 544863679@qq.com
}

Keywords: Energy Internet; energy hub; implementation plan; supporting technology

\begin{abstract}
Energy Internet, coupling energy and information deeply, is the focus of the current academic and industrial area. Meanwhile, with the cooperation of various forms of energy like electricity, gas and heat, multi-carrier energy system plays a decisive role in the development of energy internet. According to the existing international research, this paper elaborates the causes, connotation and definition of energy internet, and designs the energy internet layered architecture. With the key supporting technologies needed for the development and construction of energy internet, the complementation of multi-energy based on energy hub and its implementation scheme is proposed in energy internet, which can provide references and implementation suggestions for energy interconnection, planning operation and future energy internet construction.
\end{abstract}

\section{Introduction}

Energy crisis and environmental pollution have become increasingly serious. Multi-carrier energy system integrates electricity, heat, cooling, fuels, and transportation for collaborative analysis to increase technical, economic and environmental performance [1]. Changes in the energy sector are an important way to achieve sustainable development, and energy interconnection is the key to achieving energy change. The high permeability of large-scale distributed generation can meet future energy needs, and reduce and carbon dioxide emissions. In addition, energy storage technologies constitute a significant share of a wide range of industrial applications.

In recent years, experts and scholars have already begun a theoretical study and achieved initial results in terms of energy internet. The E-Energy initiative was launched by Germany to develop and test ICT-based energy systems [2]. The basic research framework of energy internet is established, and the basic structure and composition of energy internet are discussed. The ETH developed the concept of Energy Hub [3]. The EU launched the Future Smart Energy Internet Project, and clearly proposed energy internet development plan [4]. In China, many researchers have analyzed the global form of energy internet [5], discussed the technical connotation and characteristics [6] as well as the value and implementation architecture of energy internet [7]. The value and implementation framework are also considered. In [8], four features of energy internet are given. A generalized "Generation-Grid-Load-Storage" optimization mode of energy internet is proposed in [9]. In [10], the typical energy system including electricity/gas/heat is divided into different levers, and the integrated energy system is modeled and analyzed. However, the existing research on energy internet is only at the conception stage, and there are few international reports about the concrete implementation of energy internet.

Moving toward the implementation on a renewable-based energy hub, this paper proposes a phased implementation plan emphasizing on the integrated planning and construction of the energy layer, and summarizes the basic and key technologies in the development of energy internet. 


\section{Architecture}

In this paper, three-dimensional hierarchical structure is established to represent architecture of energy internet. According to the geographical and network structure and other factors, the system is divided into four areas including user level, local level, regional level and trans-regional level from bottom to top, which embody the coordination of generation-grid-load-storage. From the functional realization point of view, it will be divided into the energy layer, network layer, application layer and transaction layer, which represents the coordination of generation-grid-load-storage. The basic structure is shown in Figure 1.

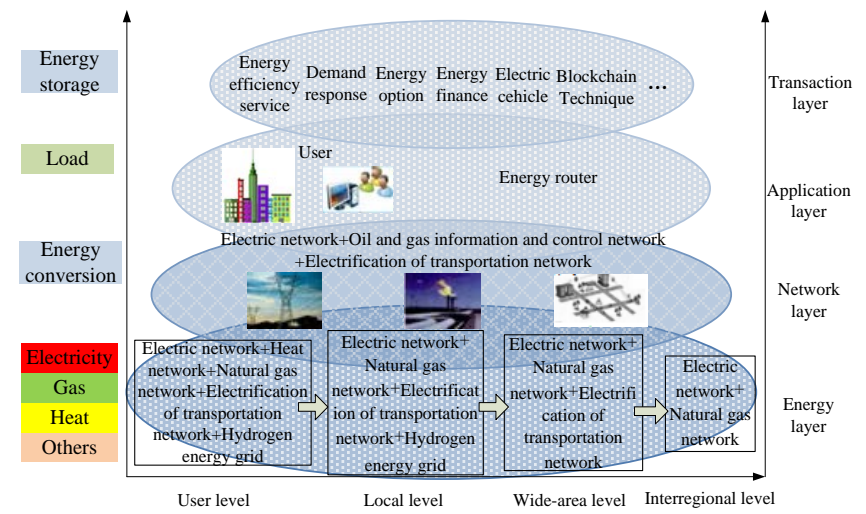

Fig.1. Hierarchical architecture of energy internet

This paper focuses on the energy interconnection analysis of the primary energy side, including energy coupling and network interconnection. From the actual construction point of view, the initial implementation path of energy internet is presented.

\section{Key Technologies}

The essence of energy internet is a highly complex physical information system, combining a number of key technologies of the energy field and information systems. According to the above layered architecture, energy internet needs energy storage, distributed power generation and other key technologies. Meanwhile, there are also several pivotal technologies that are of great importance in the implementation of energy internet, such as information and communication technology, intelligent management.

A Distributed Generation (DG) Technology

Distributed renewable energy power generation technology is the basis for energy internet in energy layer. A renewable-based generation is considered to properly model the interactions between various DG technologies, research distributed generation unit installation location, the installation capacity of a reasonable choice to make the energy internet security and economy performance optimal. The next step is to study how to achieve a seamless integration.

\section{B Energy Storage Technology}

Energy storage technology is another critical technology in energy layer, which improves the quality of power and the economic efficiency of distributed power generation unit, and maintaining stability of system. Energy storage can support the high proportion of the renewable energy power generation requirements to the multi-energy system to provide a flexible mode of operation in order to facilitate system energy management. In smart grid, energy storage has become a large-scale centralized and distributed new energy generation technology. In order to optimize the use of energy, the distributed energy storage equipment and distributed renewable energy may coordinate to adapt the widespread application of the indefinite power source.

C Information and Communication Technology

Information and communication technology is an important foundation for the realization of intelligent, interactive and large-scale power grid safe operation control in network layer. Energy Internet is a complex large scale information physical system, which can achieve the coordinated 
control and information communication. Large data will also play an important role in the planning to establish an adaptive electricity forecasting model based on large data to obtain more accurate, more fine-grained, faster time distribution and spatial distribution prediction results of power load.

D Intelligent Management

In application layer, intelligent energy management (IEM) is a hardware module for the energy internet that acts like an energy router. Through a plug-and-play interface, IEM identifies loads, calculates the price information and analyzes the availability of distributed renewable energy, and distributed energy storage devices. Intelligent fault management is a device that maintains system stability. It requires the design of a new circuit breaker to ensure that the circuit breaker can quickly and accurately isolate the fault unit when the system fails, making the solid-state transformer can quickly restore the system voltage.

E System operation control technology

The essence of energy system operation optimization in energy internet is the process of energy dispatching, which needs coordination and optimization control technology of multiple energy forms. Energy Internet system operation control technology includes multi-agent collaborative optimization technology, intelligent protection and control technology, automatic demand response technology and physical information mapping modeling analysis. By energy router, energy internet can achieve energy control, information security and network operation management.

F Blockchain technology

Blockchain can provide a de-centralization for multiple energy system of the system platform, in accordance with the development concept of energy internet. In transaction layer, blockchain technology could achieve real-time generation of electricity, energy consumption measurement, point-to-point transactions, energy use tracking, open transactions without the center and other functions. Blockchain uses asymmetric encryption algorithm to solve the trust problem between users. According to these technologies, energy internet can ensure the accuracy of information, and achieve energy system data collective maintenance and protection of user privacy.

\section{Implementation Plan}

According to the basic framework of energy internet and the key technologies, this paper puts forward a new type of energy internet implementation plan. The energy hub can be modeled as a node in a multi-energy network. The energy hubs are connected by transmission equipment, and the internal energy of the hubs can be converted to each other or stored through energy storage devices.

A Combination of electric and heat

The physical characteristics of power system and thermodynamic system are highly complementary. The distributed phase change heat storage station is regarded as a typical connecting unit of the energy network, so as to realize multi-energy interconnection and cooperative optimization. A typical energy hub is shown in Figure 2.

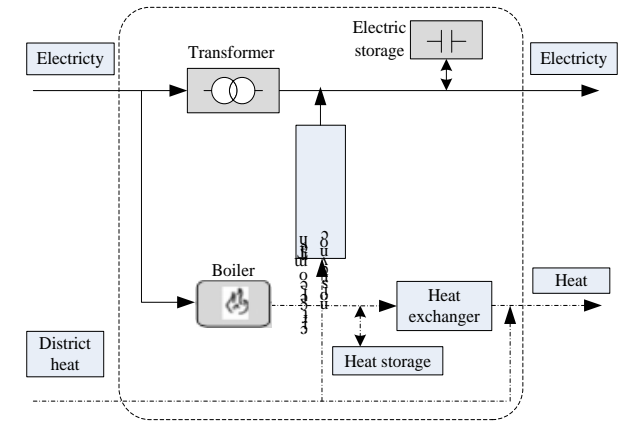

Fig.2. Electric-heat energy hub

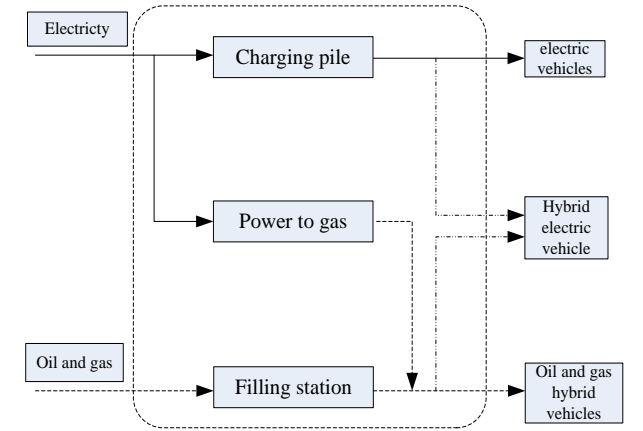

Fig.3. Electrification transportation energy hub The coupling relationship of energy supply at hour $t$ is shown as:

$\left[\begin{array}{l}L_{e}(t) \\ L_{h}(t)\end{array}\right]=C\left[\begin{array}{l}P_{e}(t) \\ P_{h}(t)\end{array}\right]-D\left[\begin{array}{l}S_{e}(t) \\ S_{h}(t)\end{array}\right]$

Where $P_{e}(t)$ and $L_{e}(t)$ are the steady-state electricity power in- and outputs respectively. 
$P_{h}(t)$ and $L_{h}(t)$ are the steady-state heat power in- and outputs respectively. $S_{e}(t)$ and $S_{h}(t)$ are the storage powers. The converter coupling matrix $C$ describes the mapping of the powers from the input to the output of the converter cluster. $\mathrm{D}$ is the storage coupling matrix.

B Electrified traffic system

Electric vehicles as a new mobile load can achieve the spatial distribution of renewable energy changes through the charging pile in electrified traffic system. Analogous to abovementioned input/output model, electrified traffic energy hub can be modeled in Figure 3. The electric vehicle battery can be effectively used to form a large-scale distributed energy storage network, and then we could achieve peak load shifting and support to renewable energy access and other functions. In the system planning stage, the layout of the charging facilities and driving and charging behavior of the owners will affect the traffic network traffic. Vehicle to Grid (V2G) technology could help the rational design of charging pile location and quantity.

C Multi-regional energy multi-network interconnection

With the availability of various of energy hubs, the interconnection of energy local area network forms a regional energy internet. The regional multi-energy system is a complex dynamic system with high dimension, multiple time series, nonlinearity and strong randomcity. The typical topology of the regional energy interconnection system is shown in Figure 4.

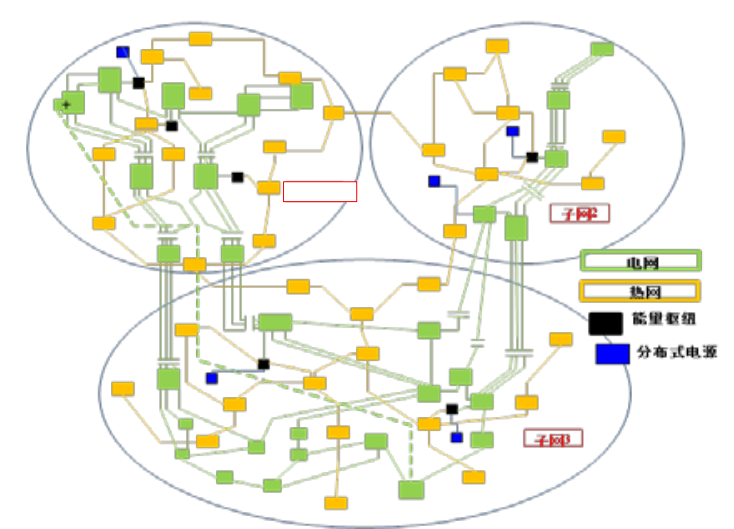

Fig.4. Topological diagram of regional energy interconnection system

Multi - regional interconnected integrated energy system takes the power transmission network and the natural gas network as the backbone network. The technology of flexible DC transmission, advanced power electronics and information physical system as the core, it mainly plays the role of energy long-distance transmission and achieves the coordination of complementary energy.

$D$ Integration of information and energy

After the realization of the interconnection of the energy network itself, implement the "Internet +", communication systems and energy network interconnection and depth of integration. The differences between the operational model and the operating habits are the main obstacles to the coordinated and unified operation of multi-energy systems. We need to break through industry barriers, confirm that service-oriented market transactions are the key to achieving multi-energy system coordination. With market transactions, cloud computing, large data and other information technology supported, the ultimately deep fusion of energy and information can be realize.

\section{CASE STUDY}

Based on the above implementation plan, coordinated wind curtailment accommodating dispatch model is proposed. The advantages of flexible power generation, high energy efficiency and environmental pollution are represented. Taking the combined heating of the residential heating network and the grid as an example proposed in Figure 2, this paper considers thermal storage boilers with maximum capacity of 10MW participating in the energy internet to build the electric-thermal energy hub and realize the energy interconnection. 


\section{A The objective function}

As discussed earlier, most of the renewable energy power generation generally has the characteristics of high investment. In order to achieve better economic benefits, we should make the electric boiler use the wind curtailment to supply heating. The objective function is as shown in (3).

$\min \Sigma_{t \in T} \Sigma_{i \in I}\left(C_{c}(i, t)+C_{f}(t)\right)$

Where $C_{c}(i, t)$ is energy cost of thermal power units $i$ in $t$ hour, $C_{f}(t)$ is punishment cost of discarding wind power at hour $t$. The coal consumption cost of thermal power units can be stated as follows:

$$
C_{c}(i, t)=\left[P^{T}(i, t)+P^{B}(i, t)\right] \times \lambda_{e, t} \times \xi_{\text {price }}^{e}
$$

Where $P^{T}(i, t), P^{B}(i, t)$ are purchased electricity energy transmitted to transformer and boiler, respectively. $\lambda_{e, t}$ is the price of electricity. $\xi_{\text {price }}^{e}$ is coefficient of electricity energy price.

$$
C_{f}(t)=C_{o} E_{F_{o}}(t)+C_{u} E_{F_{u}}(t)
$$

Where $C_{o}$ and $C_{u}$ are overestimated coefficient of penalty cost and underestimated coefficient of penalty cost, respectively. $E_{F_{O}}(t)$ and $E_{F_{u}}(t)$ are overestimated and underestimated wind energy compared to actual wind power output, respectively.

B Constraint condition

In order to make the optimal solution to the objective function, it needs to satisfy the following constraints:

$$
\begin{aligned}
& L^{(t)}=C^{(t)} P^{(t)}-S^{(t)} \dot{E}^{(t)} \\
& 0<v_{\alpha k}<1, \sum_{k} v_{\alpha k}=1 \\
& \underline{P}^{(t)} \leq P^{(t)} \leq \bar{P}^{(t)} \\
& E L N S^{\varepsilon} \leq E L N S_{U P}^{\varepsilon} \\
& L O L P^{\varepsilon} \leq L O L P_{U P}^{\varepsilon}
\end{aligned}
$$

Constraints include power balance constraints (6), energy conversion storage capacity constraints (7), converter power upper and lower constraints (8), and system reliability constraints (9) - (10). Where $E L N S^{\varepsilon}$ is the expectation load not supply of energy carrier $\varepsilon, L O L P^{\varepsilon}$ is the loss of load probability of energy carrier $\varepsilon$.

C Database

\section{1) Electrical and heat demand}

It is assumed that the typical daily load of local areas graph as shown in Figure 5.

2) Device parameters

The parameters of system are shown in Table 1.

\begin{tabular}{c|c|c}
\multicolumn{3}{c}{ Table1. System Parameters } \\
\hline \hline$\eta_{T}$ & $\eta_{B}$ & $\eta_{\text {HE }}$ \\
\hline 0.98 & 1 & 0.9 \\
\hline \hline
\end{tabular}

The thermal storage capacity of the thermal storage tank is $150 \mathrm{MWh}$, the maximum storage and heat release capacity is $40 \mathrm{MW}$.

D Calculation resuls

Through the optimal combination of three regenerative electric boilers, wind energy consumption curves are shown in Figure 6. 


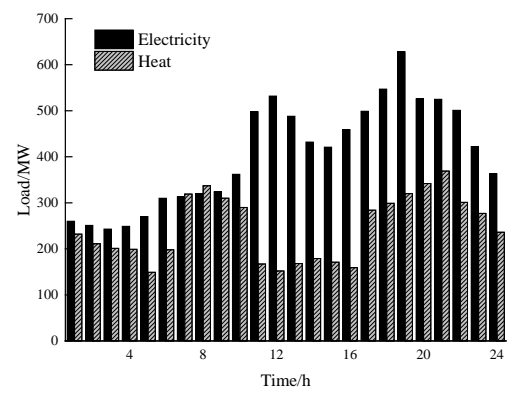

Fig.5. Electrical and heat load in a typical day

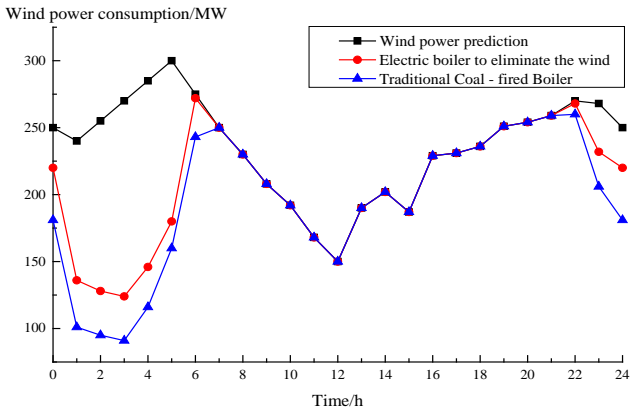

Fig.6. Wind energy consumption curves

From the calculation results, we can draw some conclusions as follow: through the regenerative electric boiler, the wind power has been effectively utilized to achieve peak load shifting, which reflect the coordination of generation and load. As the electric boiler in energy hub instead of the traditional mode of coal-fired boilers, it brings coal-saving benefits and environmental benefits.

\section{Conclusion}

By analyzing the international viewpoints of energy internet, this paper establishes three-dimensional hierarchical architecture. Based on the key technology as the foundation, we present a phased implementation plan of energy internet with electric power, heat, natural gas and transportation system as interconnection objects. In the analysis of integrated energy system, we build cross-regional energy Internet. Moreover, we integrated information and energy to analyze. By above-mentioned case study, we can obtain that energy hub has significant wind power accommodation and economic benefits. The next step will focus on design and utilization of the proper structure for the energy hubs.

\section{Acknowledgement}

This work was supported by the National Natural Science Foundation of China (61273029, 61433004), the Natural Science Foundation of Liaoning (2013020037) and the Program for New Century Excellent Talents in University, China (NCET-12-0106), the Fundamental Research Funds for the Central Universities (N130504004).

\section{References}

[1] Huang A Q, Crow M L, Heydt G T, Zheng J P, Dale S J. The Future Renewable Electric Energy Delivery and Management (FREEDM) System: The Energy Internet[J]. Proceedings of the IEEE, 2011, 99(1):133 - 148.

[2] Vermesan O, Blystad L C, Zafalon R, Moscatelli A, Kriegel K, Mock R, et al. Internet of energy-connecting energy anywhere anytime[M]. Springer Berlin Heidelberg, 2011: 33-48.

[3] Geidl M, Klokl B, Koeppel G, Andersson G, Frohlich K. Energy hubs for the futures[J]. IEEE Power \& Energy Magazine, 2007, 5(1): 24-30.

[4] European Commission. Recorded conference "mission growth: Europe at the lead of the new industrial revolution[EB/OL].(2013-1-13). http://ec.europa.eu/avservices/video/.

[5] Liu Zhenya. Global energy internet[M]. Beijing: China Electric Power Press, 2015.

[6] Zhang Xiaoping, Li Jianing, Fu Hao. Distribution Power \& Energy Internet: From Virtual Power Plants to Virtual Power Systems[J]. Proceedings of the CSEE, 2015, 35(14): 3532-3540.

[7] Yang Fang, Bai Cuifen, Zhang Yibin. Research on the Value and Implementation Framework of Energy Internet[J]. Proceedings of the CSEE, 2015, 35(14): 3495-3502. 
[8] Zha Yabing, Zhang Tao, Huang Zhuo, et al. Analysis of energy internet key technologies[J]. Science China: Information Science, 2014, 44(6): 702-713.

[9] Zeng Ming, Yang Yongqi, Liu Dun Nan, Zeng Bo, Ouyang Shaojie, Lin Haiying, et al. "Generation-Grid-Load-Storage" Coordinative Optimal Operation Mode of Energy Internet and Key Technologies[J]. Power System Technology, 2016, 40(1): 114-124.

[10]Wang Weiliang, Wang Dan, Jia Hongjie, Chen Zhaoyu, Guo Bingqing, Zhou Haiming, et al. Review of Steady-state Analysis of Typical Regional Integrated Energy System Under the Background of Energy Internet[J]. Proceedings of the CSEE, 2016, 36(12): 3292-3305. 